\title{
WESSA at SemEval-2020 Task 9: Code-Mixed Sentiment Analysis using Transformers
}

\author{
Ahmed Sultan \\ WideBot \\ a.sultan@widebot.ai \\ Amina Gaber \\ WideBot \\ amina.gaberewidebot.ai
}

\author{
Mahmoud Salim \\ WideBot \\ m.salimewidebot.ai
}

\author{
Islam EI Hosary \\ WideBot \\ islamewidebot.ai
}

\begin{abstract}
In this paper, we describe our system submitted for SemEval 2020 Task 9, Sentiment Analysis for Code-Mixed Social Media Text alongside other experiments. Our best performing system is a Transfer Learning-based model that fine-tunes XLM-RoBERTa, a transformer-based multilingual masked language model, on monolingual English and Spanish data and Spanish-English codemixed data. Our system outperforms the official task baseline by achieving a $70.1 \%$ average F1-Score on the official leaderboard using the test set. For later submissions, our system manages to achieve a $75.9 \%$ average F1-Score on the test set using CodaLab username "ahmed0sultan".
\end{abstract}

\section{Introduction}

Microblogging websites have been a huge source of data containing different kinds of information. Since the users on these microblogging websites tend to write informal real-time messages, they also tend to mix languages as they are just being spontaneous and want to ease the communication or they are just multilingual or non-native language speakers who mix between their native language and the language they are trying to use Patwa et al. (2020). This type of writing is called Code-Mixing or Code-Switching and it could be defined as the phenomenon of mixing the vocabulary and syntax of multiple languages in the same sentence Lal et al. (2019). Sentiment Analysis (SA) is the task of detecting, extracting, and classifying sentiment and opinions Montoyo et al. (2012). SA can help in structuring data of public opinions about products, brands or any topic that people can express opinions about, to be used in a very wide set of practical applications varying from political use, e.g. monitoring public events Tumasjan et al. (2010) to commercial use, e.g. making decisions in the stock market Jansen et al. (2009). The task of monolingual sentiment analysis has been a well-studied topic in the literature over the past few decades. However, little attention has been directed to SA based on code-mixed data.

In SemEval-2020 Task 9: Sentiment Analysis for Code-Mixed Social Media Text Patwa et al. (2020), the organizers provide a dataset of Code-Mixed tweets with word-level language labels that we will explore in Section 2.1, and with the following sentiment labels: positive, negative, neutral. Given a code-mixed text, the task is to classify the overall sentiment of the input text to one of the three sentiment labels mentioned above. The official evaluation metric for this task is Average F1-Score. We report the experiments made only on Spanish-English (Spanglish) data, whereas SemEval-2020 Task 9 contains Hindi-English (Hinglish) data as well. The challenges of this shared task could be summarized as follows: a) The relatively small dataset provided makes it hard to train complex models b) the target classes distribution is imbalanced in the training data $\mathrm{c}$ ) the characteristics of social media text pose difficulties such as out-of-vocabulary words and ungrammatical sentences due to the spontaneous and informal writing with lots of extra embedded information in a single sentence (e.g. hashtags, emojis, or repeated characters in a word) some of this embedded information can be utilized to make a better predictive model and others may hurt the model prediction badly.

This work is licensed under a Creative Commons Attribution 4.0 International Licence. Licence details: http: //creativecommons.org/licenses/by/4.0/. 
We conducted several experiments to tackle this problem. We used a Linear SVM, Logistic Regression, and Multinomial Naive Bayes models with TF-IDF feature vectors as an input. We also used XLMRoBERTa Conneau et al. (2020), a transformer-based multilingual masked language model which is trained on 100 languages, fine-tuned on our downstream SA task which achieves our highest score outperforming the official baseline. The rest of the paper is structured as follows: Section 2 introduces some background about the task, an overview of the dataset, and related work. We describe the applied preprocessing steps and the experiments in Section 3. We report the results in Section 4. And Section 5 summarizes our work.

\section{Background}

Whereas the task of Sentiment Analysis has been extensively explored in the past few decades, there has been less work on Code-Mixed Sentiment Analysis (CMSA). Given a sentence in the code-mixed language, the task is to predict its sentiment polarity (i.e. positive, negative, or neutral). For example, the Spanglish tweet "ha u know is true parece ke te esta dando un atake D" should be classified as a positive sentiment.

\subsection{Data}

We use a combination of external monolingual English and Spanish data, and English-Spanish code-mixed (Spanglish) data which is provided by the shared task organizers, listed below and summarized in Table 1.

- Spanglish Dataset: The dataset provided by the shared task organizers Patwa et al. (2020) consists of SpanishEnglish code-mixed tweets with target labels for each tweet, namely, positive, negative, or neutral. Also, a word-level language annotation Molina et al. (2016) was provided with the following labels (lang1, lang2, other, ne, unk, ambiguous, mixed, fw), where lang1 and lang2 tags refer to English and Spanish language respectively. The other tag refers to emojis, emoticons, usernames, URLs, symbols, and punctuation marks. The ne tag is used to label named entities. The unk tag represents all the gibberish and unintelligible tokens. Words that can be either lang 1 or lang2 are tagged as ambiguous. The mixed tag is used for code-mixed morphemes. Finally the $f w$ tag represents words from a language that is neither lang1 nor lang2. The dataset is divided into 12,002 labeled training samples, 2,998 labeled samples as development sets, and 3,789 unlabeled samples as a test set.

- T4SA Dataset: We use an external data resource to increase the training set and overcome the data imbalance in the shared task data which is a dataset of 12,000 English tweets from Twitter for Sentiment Analysis (T4SA) dataset Vadicamo et al. (2017) and we auto-translated them into 12,000 Spanish tweets using Yandex Translate API ${ }^{1}$.

\begin{tabular}{c|c|c|c|c|c|c}
\hline & \multicolumn{2}{c}{ Spanglish } & \multicolumn{2}{c}{ T4SA } & \multicolumn{2}{c}{ Total } \\
\cline { 2 - 8 } & training & development & training & development & training & development \\
\hline \hline Negative & 2023 & 506 & 8000 & NA & 10023 & 506 \\
\hline Neutral & 3974 & 994 & 8000 & NA & 11974 & 994 \\
\hline Positive & 6005 & 1498 & 8000 & NA & 14005 & 1498 \\
\hline
\end{tabular}

Table 1: Datasets Description

\subsection{Related work}

With the rise of social media data, Sentiment Analysis became a field of interest for many researchers. Read (2005) demonstrated that dependency in sentiment classification can take the form of a domain, topic, temporal and language style. To perform Sentiment Analysis of the short text Dos Santos and Gatti

\footnotetext{
${ }^{1}$ https://tech.yandex.com/translate/
} 
(2014) proposed a deep convolutional neural network that exploits information from character to sentence level. Agarwal et al. (2011) examined two types of models: tree kernel and feature-based models.

In recent years, due to the tendency of some social media users to mix two or more languages while expressing their opinions, a new code-mixed language has been generated. A lot of research has been performed on code-mixed languages, such as Hindi-English Joshi et al. (2016) and English-Spanish Solorio and Liu (2008).

Sentiment Analysis of code-mixed data received a lot of attention from the research community. Different algorithms trained on code-mixed data. Mandal and Das (2018) used supervised learning algorithms, they trained Naïve Bayes (NB) model on English features only, and then they trained Support Vector Machine (SVM) model on code-mixed data as well as English features. Patra et al. (2018) trained Support Vector Machine (SVM) model on the word and character level n-grams features. Several supervised classifiers have been tested by Mandal and Das (2018) such as Gaussian Naïve Bayes (GNB), Bernoulli Naïve Bayes (BNB) and Multinomial Naïve Bayes (MNB) from the Naïve Bayes (NB) family. In addition, they had tested Linear Models (LM): Linear Regression (LRC) and Stochastic Gradient Descent (SGDC).

Deep learning-based techniques are widely used in detecting the sentiment of code-mixed data. Konate and $\mathrm{Du}$ (2018) showed that deep learning models outperformed the classical algorithms as deep learning models introduce more non-linearity in feature space, they tested with different models and their best model was one-layer Convolutional Neural Network (CNN). A sophisticated method utilizes the shared parameters of Siamese networks to map the sentences of code-mixed and standard languages to a common sentiment space that has been tested by Choudhary et al. (2018). A sub-word level LSTM (SubwordLSTM) architecture for learning sentiments in code-mixed data has been utilized in Joshi et al. (2016). Another line of research, Ghosh et al. (2017) they created their own dataset by manually labeling Facebook posts with their associated sentiments. They used word-based, semantic, and style-based features for classification. A Multilayer Perceptron model has been used to determine the polarity of the sentiment.

\section{System Description}

In this section, we are proposing different approaches to tackle the problem of identifying the sentiment in code-mixed text.

\subsection{Preprocessing}

As social media text is different from the regular text with irregular grammar, elongation, and all the informal usages of language, we needed to improve the quality of the text as it has a major effect on the final score. So we perform our preprocessing steps as follows:

- Remove emoticons and emojis and replace them with their textual meaning.

"I love you so much $\odot<3$ " $\rightarrow$ "I love you so much smiley face heart"

- Remove Mentions and non-ASCII Characters.

- Replace URLs with <URL> "The article URL is www.example.com" $\rightarrow$ "The article URL is URL"

- Remove elongation. "Hiiiii everyone" $\rightarrow$ "Hi everyone"

- Extract words from hashtags. "We need to talk \#HereWeGoAgain" $\rightarrow$ "We need to talk Here We Go Again"

\subsection{TF-IDF Vectorization}

For the purpose of fitting the TF-IDF vectorizer, we used two different methods for preparing the input data:

- We treated each sentence in the datasets as a document so that we have input data size with the same size of the datasets mentioned in Section 2.1.

- For each sentiment label, we concatenated all the text so that we have an input of three rows representing the three sentiments (positive, neutral, negative). 
For each one of the approaches mentioned above, we fitted two TF-IDF vectorizers on the whole text, the first vectorizer is a word-level TF-IDF vectorizer, and the second one is a character level TF-IDF vectorizer, then we concatenate the two vectorizers outputs into one vector which will be the input for the models discussed in Section 3.3 as illustrated in Figure1.

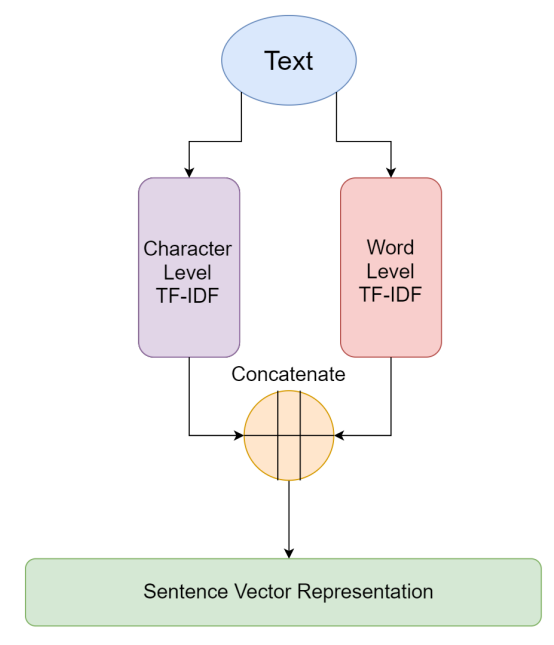

Figure 1: TF-IDF vectorization process

\subsection{Machine Learning (ML) Models}

We experimented with three different classical ML models: Logistic Regression (LR), Multinomial Naive Bayes (MNB), and Support Vector Machine (SVM) with a linear kernel Cortes and Vapnik (1995). Each model is trained on either the task dataset only or the T4SA data combined with the task dataset using the TF-IDF vectorization techniques explained in Section 3.2.

After several experiments with different settings, we found that increasing the train data size with the T4SA dataset doesn't have a noticeable increase in the evaluation metric perhaps that's because this dataset is from a different distribution which is monolingual, unlike the task dataset which is mostly code-mixed. Another reason could be the error propagation that might happen due to the automatic translation process, from English to Spanish, on the external dataset. So we only report the experiments conducted using the task dataset.

\subsection{XLM-RoBERTa}

Our best performing system is a fine-tuned XLM-RoBERTa Conneau et al. (2020), which is a generalpurpose sentence representation and an extended version of mBERT and XLM (Lample and Conneau, 2019; Devlin et al., 2018) As they trained a transformer model Vaswani et al. (2017), with the multilingual Masked Language Model (MLM) objective using only monolingual data of 100 languages, on more data using Common-Crawl rather than using mere Wikipedia text. Using HuggingFace Transformers library Wolf et al. (2019), We fine-tuned XLM-RoBERTa base model on both Spanglish and T4SA training data in a randomly shuffled fashion. The preprocessing mentioned in Section 3.1 is used excluding 2 steps: the removal of non-ASCIIcharacters and word extraction from hashtags. This model is the submitted version which achieved a $70.1 \%$ average F1-Score on the official test set leaderboard.

Another version of the model described above, with slight changes in model's hyperparameters and preprocessing, achieved a $75.9 \%$ average F1-Score on the ofcial leaderboard test set. But due to the task limit number of submissions, this model wasn't considered on the official leaderboard.

We even enhance this model further after the submission phase by training the model on the monolingual data first then the code-mixed data Choudhury et al. (2017) achieving a higher score than its preceding versions on the development set rather than the test set since the test labels are never released. 


\section{Results}

\begin{tabular}{|c|c|c|}
\hline System & TF-IDF Input & Dev Avg F1-Score \\
\hline \hline LR & $\mid$ concatenated docs per class & $51.60 \%$ \\
\cline { 2 - 3 } MNB & all documents & $49.60 \%$ \\
\hline SVM & $\mid$ concatenated docs per class & $50.40 \%$ \\
\cline { 2 - 3 } & all documents & $50.73 \%$ \\
\hline $\begin{array}{c}\text { XLM-RoBERTa } \\
\text { (submitted) }\end{array}$ & all documents & $51.53 \%$ \\
\hline $\begin{array}{c}\text { XLM-RoBERTa } \\
\text { (enhanced) }\end{array}$ & NA & $\mathbf{5 2 . 2 0 \%}$ \\
\hline
\end{tabular}

Table 2: Evaluation results on development set

In this section, We report the results of our experiments in SemEval 2020 Task 9, Sentiment Analysis for Code-Mixed Social Media Text which introduces different systems, preprocessing steps, and data sources used. The official evaluation metric used is Average F1-Score. Due to the lack of test set labels, only the submitted system has a test set score, so we compare the different systems based on the development set scores. Table 2 shows the performance of all the systems described in the previous section. Compared with the other systems, the enhanced XLM-RoBERTa system achieves the highest score with a $54.74 \%$ average F1-Score on the development set outperforming the submitted XLM-RoBERTa system which achieved only $52.20 \%$.

\section{Conclusion}

In this paper, we described our experimentations on SemEval 2020 Task 9, Sentiment Analysis for Code-Mixed Social Media Text. We presented four main systems; Logistic Regression, Multinomial Naive Bayes, SVM with linear kernel, and a fne-tuned XLM-RoBERTa. The submitted XLM-RoBERTa system outperformed the baseline on the test set with an average F1-Score of $70.1 \%$ and $52.20 \%$ on the development set. Whereas the enhanced approach of the XLMRoBERTa system outperformed the submitted version by achieving a 54.7\% average F1-Score on the development set.

Future work includes working on using multilingual word embeddings that's aligned in the same space like MUSE Lample et al. (2018).

\section{References}

Apoorv Agarwal, Boyi Xie, Ilia Vovsha, Owen Rambow, and Rebecca Passonneau. 2011. Sentiment analysis of twitter data. In Proceedings of the Workshop on Languages in Social Media.

Nurendra Choudhary, Rajat Singh, Ishita Bindlish, and Manish Shrivastava. 2018. Sentiment Analysis of CodeMixed Languages leveraging Resource Rich Languages.

Monojit Choudhury, Kalika Bali, Sunayana Sitaram, and Ashutosh Baheti. 2017. Curriculum Design for Codeswitching: Experiments with Language Identification and Language Modeling with Deep Neural Networks. Proceedings of the 14th International Conference on Natural Language Processing (\{ICON\}-2017), pages 6574.

Alexis Conneau, Kartikay Khandelwal, Naman Goyal, Vishrav Chaudhary, Guillaume Wenzek, Francisco Guzmán, Edouard Grave, Myle Ott, Luke Zettlemoyer, and Veselin Stoyanov. 2020. Unsupervised Cross-lingual Representation Learning at Scale. In Proceedings of ACL 2020.

Corinna Cortes and Vladimir Vapnik. 1995. Support-Vector Networks. Machine Learning.

Jacob Devlin, Ming-Wei Chang, Kenton Lee, and Kristina Toutanova. 2018. BERT: Pre-training of Deep Bidirectional Transformers for Language Understanding. In NAACL HLT 2019 - 2019 Conference of the North American Chapter of the Association for Computational Linguistics: Human Language Technologies - Proceedings of the Conference (Vol. 1, pp. 4171-4186), (Mlm). 
Cícero Nogueira Dos Santos and Maíra Gatti. 2014. Deep convolutional neural networks for sentiment analysis of short texts. In COLING 2014 - 25th International Conference on Computational Linguistics, Proceedings of COLING 2014: Technical Papers, pages 69-78. Association for Computational Linguistics, ACL Anthology.

Souvick Ghosh, Satanu Ghosh, and Dipankar Das. 2017. Sentiment Identification in Code-Mixed Social Media Text.

Bernard J. Jansen, Mimi Zhang, Kate Sobel, and Abdur Chowdury. 2009. Twitter power: Tweets as electronic word of mouth. Journal of the American Society for Information Science and Technology.

Aditya Joshi, Ameya Prabhu, Manish Shrivastava, and Vasudeva Varma. 2016. Towards sub-word level compositions for sentiment analysis of Hindi-English code mixed text. In COLING 2016 - 26th International Conference on Computational Linguistics, Proceedings of COLING 2016: Technical Papers, pages 2482-2491. Association for Computational Linguistics, ACL Anthology.

Arouna Konate and Ruiying Du. 2018. Sentiment Analysis of Code-Mixed Bambara-French Social Media Text Using Deep Learning Techniques. Wuhan University Journal of Natural Sciences, 23(3):237-243.

Yash Kumar Lal, Vaibhav Kumar, Mrinal Dhar, Manish Shrivastava, and Philipp Koehn. 2019. De-Mixing Sentiment from Code-Mixed Text. In In ACL 2019 - 57th Annual Meeting of the Association for Computational Linguistics, Proceedings of the Student Research Workshop (pp. 371-377).

Guillaume Lample and Alexis Conneau. 2019. Cross-lingual Language Model Pretraining.

Guillaume Lample, Alexis Conneau, Marc'Aurelio Ranzato, Ludovic Denoyer, and Hervé Jégou. 2018. Word translation without parallel data. In 6th International Conference on Learning Representations, ICLR 2018 Conference Track Proceedings.

Soumil Mandal and Dipankar Das. 2018. Analyzing Roles of Classifiers and Code-Mixed factors for Sentiment Identification. 1.

Giovanni Molina, Fahad AlGhamdi, Mahmoud Ghoneim, Abdelati Hawwari, Nicolas Rey-Villamizar, Mona Diab, and Thamar Solorio. 2016. Overview for the Second Shared Task on Language Identification in Code-Switched Data. In Association for Computational Linguistics (ACL).

Andrés Montoyo, Patricio Martínez-Barco, and Alexandra Balahur. 2012. Subjectivity and sentiment analysis: An overview of the current state of the area and envisaged developments. Decision Support Systems, 53(4):675679.

Braja Gopal Patra, Dipankar Das, and Amitava Das. 2018. Sentiment Analysis of Code-Mixed Indian Languages: An Overview of SAIL_Code-Mixed Shared Task @ICON-2017.

Parth Patwa, Gustavo Aguilar, Sudipta Kar, Suraj Pandey, Srinivas PYKL, Björn Gambäck, Tanmoy Chakraborty, Thamar Solorio, and Amitava Das. 2020. SemEval-2020 Task 9: Overview of Sentiment Analysis of CodeMixed Tweets. In Proceedings of the 14th International Workshop on Semantic Evaluation (SemEval-2020), Barcelona, December. Association for Computational Linguistics.

Jonathon Read. 2005. Using emoticons to reduce dependency in machine learning techniques for sentiment classification. In ACL-05 - 43rd Annual Meeting of the Association for Computational Linguistics, Proceedings of the Conference.

Thamar Solorio and Yang Liu. 2008. Part-of-Speech tagging for English-Spanish code-switched text. In EMNLP 2008 - 2008 Conference on Empirical Methods in Natural Language Processing, Proceedings of the Conference: A Meeting of SIGDAT, a Special Interest Group of the ACL, pages 1051-1060.

Andranik Tumasjan, Timm O. Sprenger, Philipp G. Sandner, and Isabell M. Welpe. 2010. Predicting elections with Twitter: What 140 characters reveal about political sentiment. In ICWSM 2010 - Proceedings of the 4th International AAAI Conference on Weblogs and Social Media, pages 178-185. Word. Journal Of The International Linguistic Association.

Lucia Vadicamo, Fabio Carrara, Andrea Cimino, Stefano Cresci, Felice Dell'Orletta, Fabrizio Falchi, and Maurizio Tesconi. 2017. Cross-Media Learning for Image Sentiment Analysis in the Wild. In Proceedings - 2017 IEEE International Conference on Computer Vision Workshops, ICCVW 2017.

Ashish Vaswani, Noam Shazeer, Niki Parmar, Jakob Uszkoreit, Llion Jones, Aidan N. Gomez, Łukasz Kaiser, and Illia Polosukhin. 2017. Attention is all you need. In Advances in Neural Information Processing Systems, volume 2017-Decem, pages 5999-6009.

Thomas Wolf, Lysandre Debut, Victor Sanh, Julien Chaumond, Clement Delangue, Anthony Moi, Pierric Cistac, Tim Rault, Rémi Louf, Morgan Funtowicz, and Jamie Brew. 2019. HuggingFace's Transformers: State-of-theart Natural Language Processing. 